\title{
Impurity photoconduction, excitons, and effective masses in liquid xenon
}

\author{
M. Reshotko and U. Asaf \\ Racah Institute of Physics, The Hebrew University, Jerusalem, Israel 91904
}

G. Ascarelli

Department of Physics, Purdue University, West Lafayette, Indiana 47097

R. Reininger

Hasylab at DESY, Notkestrasse 85, 2000 Hamburg 52, Federal Republic of Germany

\author{
G. Reisfeld and I. T. Steinberger
}

Racah Institute of Physics, The Hebrew University, Jerusalem, Israel 91904

(Received 31 July 1990; revised manuscript received 30 November 1990)

\begin{abstract}
Photoconductivity excitation spectra in liquid xenon doped by methyl iodide or toluene were recorded for a broad range of impurity concentrations $(5 \mathrm{ppm}-1.1 \%)$ and xenon densities ranging from the critical point to the triple point. Electrons liberated from the impurity molecules yield photocurrent in a broad spectral range, with excitonic transitions of the xenon host appearing as dips on a continuous background. It is demonstrated that, with the experimental conditions employed, the reciprocal values of the photocurrent represent fairly well the absorption spectrum of the host. Such reciprocal spectra enabled us to determine the parameters of the $n=2$ Mott-Wannier exciton for densities $\rho$ ranging from 9.5 to $13.6 \times 10^{21} \mathrm{~cm}^{-3}$. From these, in turn, along with previously determined intrinsic photoconduction threshold values, the electron effective mass $m_{e}$ was determined as $\approx 0.28 m$ ( $m$ being the free-electron mass) in the whole range of densities, with indication for a shallow broad minimum at $\rho \approx 11 \times 10^{21} \mathrm{~cm}^{-3}$.
\end{abstract}

\section{INTRODUCTION}

Studying electron states by optical means in a highly absorbing liquid is wrought with difficulties. If the vapor pressure of the liquid is not exceptionally low, it has to be investigated in a closed cell; the light enters the cell after passing through an optical window. This prevents the use of modern electron energy analysis methods. A high absorption coefficient necessitates the use of very thin absorption cells: if the imaginary part $k$ of the refractive index is of the order of unity and the spectra of interest are in the vacuum-ultraviolet region, optical path lengths have to be impractically small, of the order of $10^{-6}-10^{-5}$ $\mathrm{cm}$. Reflection spectra are again hampered by the need of an optical window; nearness of the refractive indices of the window material and that of the liquid reduces the measured reflectance. Though each of the methods mentioned has been used with considerable success by dedicated research teams, a simple additional method for obtaining an overview of the optical properties, especially the absorption coefficient $\alpha$ (and $k$ ) can be rather useful. We show in this paper that the reciprocal impurity photocurrent can give, under appropriate circumstances, an approximate absorption spectrum.

Among the liquids, those of the rare gases have a special role. It has been shown that the electron mobility in these materials is very large, comparable to that in the respective solids. ${ }^{1-5}$ Excitons, both of Wannier-Mott and "intermediate" type, have been reported in pure ${ }^{6,10}$ as well as doped ${ }^{11-13}$ rare-gas liquids. The difference between atomic levels shifted and broadened by molecular interactions with neighboring atoms on one hand and the intermediate excitons on the other has been clearly demonstrated and shown to be associated with density fluctuations within the liquid. 8,10 The $n=2$ exciton appears at densities higher than the minimum density needed for the appearance of the $n=1$ "intermediate" exciton; ${ }^{9}$ this fact was attributed to the extended size of the former. While the existence of these Mott-Wannier excitons in the rare-gas liquids underlines the similarities between the electronic states of these liquids with the corresponding solids, no data have been published on the dependence of the exciton parameters - its oscillator strength, position, and width-on the density. This is due to the fact that in the reflection spectra this exciton appears as a weak shoulder superimposed on the flank of the much stronger $n^{\prime}=1$ "intermediate" exciton. A very notable exception is the reflection spectrum obtained at two different angles of incidence by Subtil et al. ${ }^{14}$ in the liquid and in the solid near the triple point. From these measurements the authors obtained, by direct calculations, a complete and reliable set of optical constants, containing, of course, all the information about the $n=2$ Mott-Wannier excitons as well.

In principle, knowledge of the position of the $n=2$ Mott-Wannier exciton coupled with the determination of the band gap should yield the reduced effective exciton mass $m_{\mathrm{exc}} / m$ (in terms of the free-electron mass $m$ ), pro- 
vided the dielectric constant $\epsilon$ is known. If there exist estimates for the hole effective mass and if this is much larger than the electron effective mass, good estimates can be made for the electron effective mass $m_{e} . m_{e}$, in turn, is a very important parameter used in theories on the density dependence of the electron mobility.

In this paper we present spectra showing the photon energy dependence of the extrinsic photocurrent in liquid xenon, i.e., photocurrent due to electrons raised from isolated impurity molecules into the conduction states ("conduction band") of the host. The spectra were obtained for a broad range of densities and impurity concentrations. Excitonic transitions of the xenon host below the xenon band gap appear as dips on the impurity photoconductivity excitation spectra, since these transitions do not lead to photoconduction (except by eventual excitation transfer) and they compete with the impurity for the incident photons. Under clearly definable conditions, the reciprocal of the photocurrent is a linear function of the absorption coefficient $\alpha_{m}$ of the xenon host. These reciprocal spectra are easily analyzed. In particular, good data are obtained for the parameters of the $n=2$ Mott-Wannier exciton for xenon. For the first time in any liquid, such exciton parameters are presented in a broad density range $\left[(9.5-13.6) \times 10^{21} \mathrm{~cm}^{-3}\right]$. In particular, the reduced effective mass of the exciton $m_{\text {exc }} / m$ is calculated and presented. $m_{\mathrm{exc}} / m$ is roughly constant in the density range mentioned, with indications for a shallow minimum around $11 \times 10^{21} \mathrm{~cm}^{-3}$, near the position of the well-known minimum of the lowest energy $V_{0}$ of a conduction electron. ${ }^{15}$

\section{EXPERIMENT}

All the experiments were performed at HASYLAB using synchrotron radiation monochromated by means of the HONORMI system. ${ }^{16}$ The experimental cell was built of a hollow 36-mm stainless-steel cube equipped with four ports. Standard ultrahigh vacuum CF flanges mounted (by means of copper gaskets) on the ports carried the following items: (i) two low-current electrical feedthroughs, (ii) a $\mathrm{MgF}_{2}$ entrance window glued to the flange by a low-vapor pressure epoxy resin, ${ }^{17}$ (iii) the filling tube, and (iv) a small copper tube reaching almost to the center of the cube, into which a snugly fitting platinum resistor was inserted for temperature measurements. Two parallel gold electrodes were deposited by sputtering on the inner surface of the $\mathrm{MgF}_{2}$ window before assembly. Light reached the contents of the cell (doped xenon) through the gap $(1 \mathrm{~mm})$ between the electrodes. The gold electrodes were connected electrically to the feedthroughs by means of indium-plated screw heads, pressed unto the electrodes by means of springs. This arrangement ensured trouble-free contacts in the whole temperature and pressure range. dc voltages were applied, usually of $300 \mathrm{~V}$. The temperature was controlled to an accuracy better than $1^{\circ} \mathrm{C}$. Densities were determined by means of the set of isotherms by Streett, Sagan, and Staveley. ${ }^{18}$

Xenon gas from Deutsche 1'Air Liquide, 99.99\% purity, was used, without further purification. The dopants were analytic grade methyl iodide or toluene; in some preliminary measurements the dopant was benzene. The dopant concentration was varied between $5 \mathrm{ppm}$ and $1.1 \%$. The results presented here are part of an extensive series on photo-conduction in fluid xenon, including the gas phase as well. ${ }^{19}$

\section{RESULTS}

Figure 1 represents the photoconductivity excitation spectrum of a series of liquid xenon samples doped by $1.1 \%$ of methyl iodide molecules, at various liquid densities. The current $i\left(10^{-14}-10^{-10} \mathrm{~A}\right)$ is normalized to equal numbers of incident photons; this normalization is justified in view of the observed proportionality between the current and the number of incident photons. It is seen that in this density range the impurity photoconduction starts at around $7.7 \mathrm{eV}$. Between $\approx 8.1$ and $8.4 \mathrm{eV}$ a broad depression appears consisting of two neighboring dips. Their positions are denoted by $E_{1}$ and $A$, respectively. $E_{1}$ is approximately at the energy of the "intermediate" exciton in liquid xenon, and $A$ at about the adjoining vestigial $6 s(3 / 2)_{1}$ atomic line. ${ }^{8}$ Beyond this double dip the current increases, followed again by a descent to a minimum (denoted by $E_{1}^{\prime}$ ) around $9.4 \mathrm{eV}$, corresponding to the $n^{\prime}=1$ intermediate exciton. On the decreasing slope of this minimum a further depression (denoted by $E_{2}$ ) is seen, around $9 \mathrm{eV}$; this is the approximate position of the $n=2$ Mott-Wannier exciton in pure

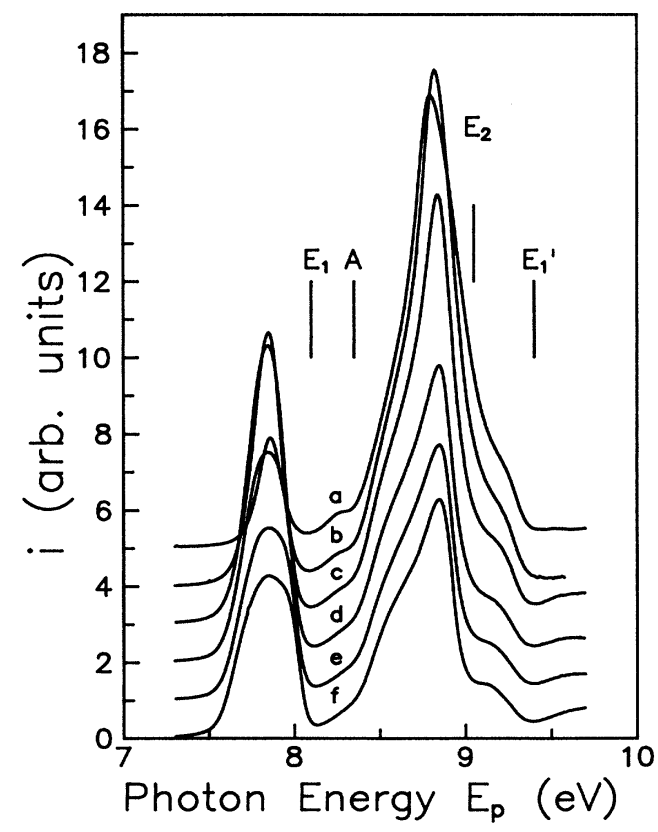

FIG. 1. Photocurrent normalized for equal numbers of incident photons $i$, as a function of photon energy $E_{p}$, in $\mathrm{Xe}$ doped with $1.1 \% \mathrm{CH}_{3} \mathrm{I}$. The spectra are staggered uniformly. Number densities: curve $a, 0.996 \times 10^{22}$; curve $b, 1.11 \times 10^{22}$; curve $c, 1.20 \times 10^{22}$; curve $d, 1.28 \times 10^{22}$; curve $e, 1.33 \times 10^{22}$; curve $f, 1.36 \times 10^{22}$. 
liquid xenon. ${ }^{7,14}$ The qualitative interpretation of the structures mentioned is straightforward: the photocurrent is due to electrons liberated by the photons from the discrete dopant levels into the quasifree states ("conduction band"). On the other hand, absorption of photons of energies $E_{1}, A, E_{1}^{\prime}$, and $E_{2}$ by the xenon host does not involve photoconduction. ${ }^{20}$ Thus these excitations compete with the impurity for the incident photons, creating dips in the spectra. Such dips are characteristic features of impurity photoconduction; their observation and analysis is the central subject of this work.

It should be noted in passing that at very low impurity concentrations ( $\leq 50 \mathrm{ppm}$ ) peaks appear instead of dips at the energies $E_{1}, A, E_{1}^{\prime}$, and $E_{2}$ (not shown). We attribute this behavior to an excitation transfer process from the excitons either to the electrodes, or to the windows or to the impurity: such processes become prominent only when the impurity photocurrents are small because of the low concentration of impurity molecules.

The slight rise of the curves beyond $9.4 \mathrm{eV}$ is partly due to the onset of intrinsic photoconduction [near the triple point in pure samples at about $9.3 \mathrm{eV}$ (Ref. 20)]. The intrinsic photocurrent is superimposed on the impurity photocurrent: in samples with lower doping levels than shown this rise is more prominent and starts nearer to $9.3 \mathrm{eV}$ (cf., Fig. 3 and its discussion below). The intrinsic photocurrents observed are relatively weak since there is a strong competition for the incident photons by the exciton denoted by $E_{1}^{\prime}$. In this context we wish to draw the attention to the fact that even in pure liquid xenon the onset of band-to-band excitation of electrons could never be observed in optical absorption or reflection spectra, ${ }^{6-9}$ but only in photoconductivity excitation spectra. $^{20}$

Figure 2 shows $1 / i E_{p}$, i.e., the reciprocal values of the data of Fig. 1 divided by the photon energy $E_{P}$, as a function of $E_{P}$. The dips of Fig. 1 appear now as peaks. It will be shown (see Discussion) that $1 / i E_{P}$ is proportional to the imaginary part $k$ of the complex refractive index of the xenon host, provided the following conditions are met.

(i) There is no intrinsic photoconduction, i.e., no direct excitation of electrons from filled bands of the host into the conduction band.

(ii) The excitonic excitations of the host do not cause photoconduction indirectly, namely, by excitation transfer from the $\mathrm{Xe}$ host either to the impurity molecules, to the electrodes, or to the window. As stated above, in our experiments energy transfer became significant only at very low impurity concentrations $(<50$ ppm).

Conditions 1 and 2 taken together mean that the photocurrents are entirely due to raising electrons from the impurity levels into the conduction band of the host liquid.

(iii) The light at each wavelength is totally absorbed within a depth where the field variation is negligible [see Eq. (3) in the Discussion]. In the experiments presented the absorption coefficient is always larger than $10^{3} \mathrm{~cm}^{-1}$, the interelectrode distance is $0.1 \mathrm{~cm}$ and the electrode thickness is of the order of $1 \mu \mathrm{m}$. Under these circumstances the condition is met everywhere except in a

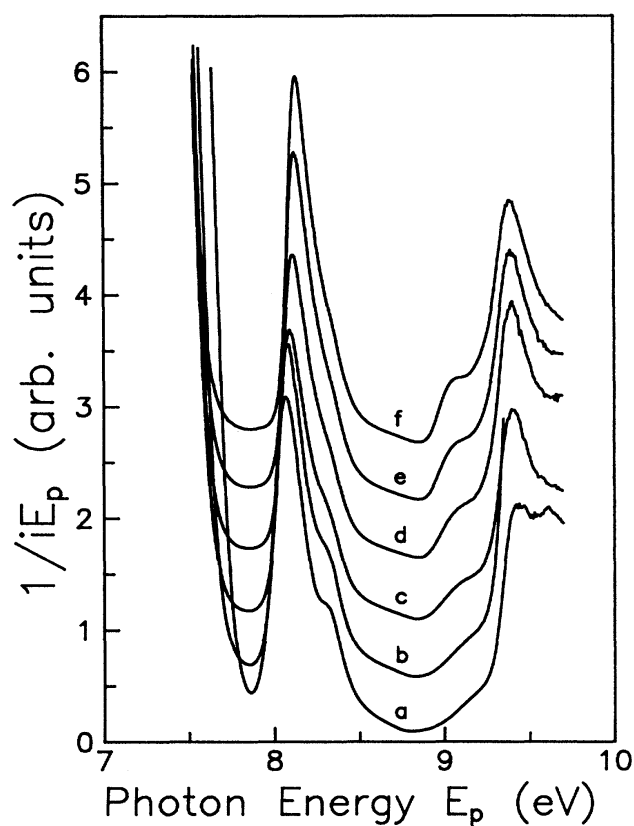

FIG. 2. $1 / i E_{p}, i$ being the photocurrent and $E_{p}$ the photon energy as a function of $E_{p}$ in Xe doped with $1.1 \% \mathrm{CH}_{3} \mathrm{I}$. The spectra are staggered uniformly. Number densities same as in Fig. 1.

region within a distance of about $20 \mu \mathrm{m}$ from electrodes. The contributions to the current of these regions where the field is also appreciably higher than midway between the electrodes are not negligible. However, from the value of the saturation electron drift velocity of xenon $\left(\approx 10^{5} \mathrm{~cm} / \mathrm{sec}\right)$ (Ref. 4) and the estimated lifetime $\left(10^{-8}-10^{-7} \mathrm{sec}\right.$, see also Appendix $)$ one sees that each electron travels a distance of 10 to $100 \mu \mathrm{m}$ before its recombination. Moreover, at the peaks of Fig. 2 (corresponding to the minima of Fig. 1) the absorption coefficient is much larger than $10^{3} \mathrm{~cm}^{-1}$ and quantitative conclusions of this paper refer only to such spectral regions. Thus the conduction is met in most of the interelectrode space and its violation near the electrodes is of no consequence.

(iv) In the spectral region and at the concentrations involved the absorption coefficient $\alpha_{p}$ of the impurity is constant at any given concentration and small compared with that of the host $\alpha_{m}$.

(v) The lifetime $\tau$ of photoelectrons is independent of photon energy. This follows from the observed proportionality between the current and number of incident photons. The lifetime is also independent of the distance from the entrance window at which the free electron was created; for example, there are no "dead layer" effects at the window. Though such effects are common, we did not observe them in our experiments.

Before discussing the degree of validity of assumptions (i) and (iv), we compare some of our data displayed as in Fig. 2 (i.e., $1 / i E_{p}$ ) with the best results available on $k$ in liquid and solid xenon, obtained by Subtil et al. ${ }^{14}$ These 
authors took reflectivity spectra of pure liquid and solid xenon near the triple point at two angles of incidence, ${ }^{14}$ namely, at $2.2^{\circ}$ and $69.4^{\circ}$. The reflecting surface was the interface of liquid xenon and the $\mathrm{MgF}_{2}$ entrance window. From the reflectivities obtained at two angles they calculated $n$ and $k$ by solving the Fresnel equations.

Figure 3 shows a comparison of some of our spectra with the absorption spectrum obtained by Subtil et al. ${ }^{14}$ All data were taken near the triple point. In order to facilitate the comparison we normalized the $1 / i E_{p}$ spectra so that the height of the $E_{1}$ peak was equal to the height of this peak in the $k$ versus $E_{p}$ spectrum. The graphs of Fig. 2 were normalized in the same way: the normalization factor was found from the comparison of peak $E_{1}$ of the spectrum ef with the same peak in the spectrum by Subtil et al. ${ }^{14}$ It is seen in Fig. 3 that all the features of the $k$ versus $E_{p}$ spectrum appear in the $1 / i E_{p}$ spectra as well, namely, the peaks $E_{1}, E_{2}$, and $E_{1}^{\prime}$ (the peak $A$ is only a small shoulder at this density in both kinds of spectra). However, there are several differences, the most obvious one being the considerably smaller height of the $E_{1}^{\prime}$ peak in our spectra in comparison with the $k$ versus $E_{p}$ spectrum. ${ }^{14}$ The reason for this is obvious, if we take into account that in the triple point liquid the onset of intrinsic photoconduction (band-to-band transitions) is at $\approx 9.3 \mathrm{eV} .{ }^{20}$ Thus condition (i) is violated in the spectral region discussed. The intrinsic photocurrent adds to the extrinsic one, reducing considerably the values of $1 / i E_{p}$.

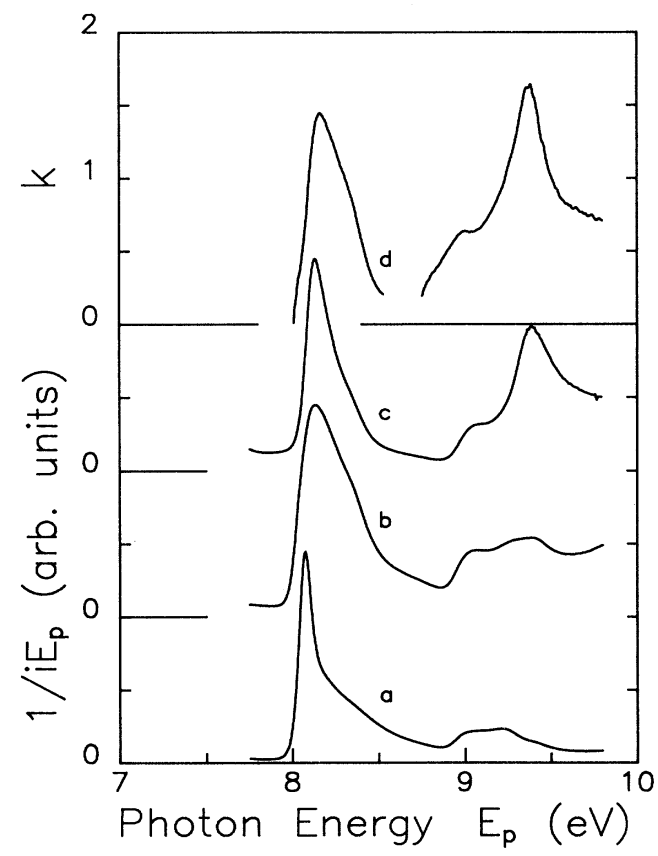

FIG. 3. Comparison of $1 / i E_{p}$ and the absorption coefficient $k$ of pure xenon as measured by Subtil et al. (Ref. 14). Our data $(a, b$, and $c)$ are normalized to that of Subtil et al. (curve $d$ ). The number density is $1.36 \times 10^{22} \mathrm{~cm}^{-3}$ in all spectra. Doping concentrations are as follows: curve $a, \mathrm{CH}_{3} \mathrm{I} 125 \mathrm{ppm}$; curve $b$, toluene 600 ppm; curve $c, \mathrm{CH}_{3} \mathrm{I} 1.1 \%$; curve $d$, pure xenon.

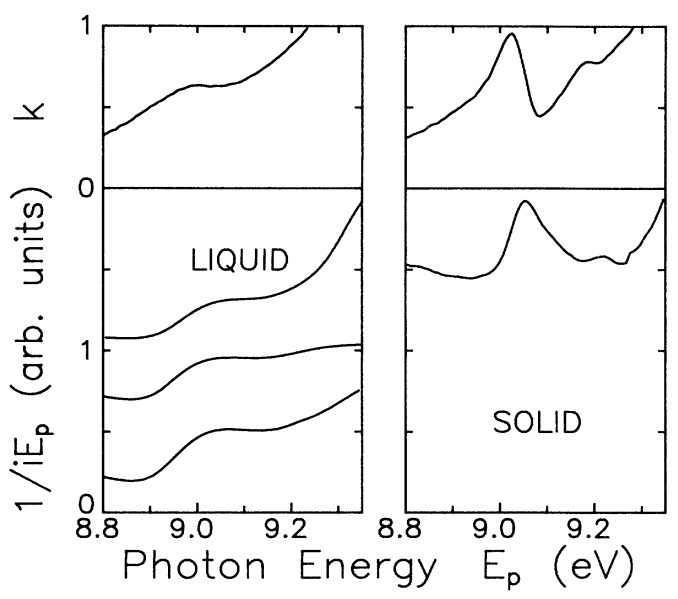

FIG. 4. Comparison of $1 / i E_{p}$ and the absorption coefficient $k$ of pure xenon (as in Fig. 3) in both liquid and solid phases in the region of the $n=2$ exciton peak. Liquid: From bottom to top, staggered vertically: Toluene $6000 \mathrm{ppm}, 1.35 \times 10^{22} \mathrm{~cm}^{-3}$; Toluene $600 \mathrm{ppm}, 1.36 \times 10^{22} \mathrm{~cm}^{-3} ; \mathrm{CH}_{3} \mathrm{I} 1.1 \%, 1.36 \times 10^{22}$ $\mathrm{cm}^{-3}$. Solid: Toluene $25 \mathrm{ppm}, 1.55 \times 10^{22} \mathrm{~cm}^{-3}$.

This interpretation is strongly supported by the fact that the $E_{1}$ peak in our spectra decreases with the decrease of the impurity concentration: the smaller the impurity concentration the higher the relative contribution of the intrinsic photocurrent.

Smaller differences between the two spectra, especially shifts and some further structure appearing in the $1 / i E_{p}$ versus $E_{p}$ spectra can be attributed to the violation of condition (iv). In fact, pure methyl iodide has several peaks in the region, but for the high density of perturbers of the present work they can be expected to merge ${ }^{21}$ into a single broad band centered at about $7.7 \mathrm{eV}$. The unusual shape of the $E_{1}$ peak in graph Fig. 3(a) might be due to this effect.

Figure 4 shows in more detail the spectral region around $9 \mathrm{eV}$ in several $1 / i E_{p}$ versus $E_{p}$ spectra near the triple point for both liquid and solid xenon, in comparison with the $k$ versus $E_{p}$ spectra by Subtil et al. This is the region of the Mott-Wannier excitons. It is seen that the exciton peak appears very clearly, though shifted to the blue when compared with its position in the $k$ versus $E_{p}$ spectra. The shift is due to the high impurity concentration: this point will be discussed below. The width and height of the $E_{2}$ peak in our $1 / i E_{p}$ spectra are very similar to those of the $k$ spectra.

\section{DISCUSSION}

\section{A. The reciprocal current representation}

The results presented above indicate that plotting $1 / i E_{p}$ spectra ( $i$ being the impurity photocurrent), is a useful way to obtain approximate absorption spectra of the host for appropriate host-impurity combinations. We will show now that this presentation is indeed valid: $1 / E_{p}$ is proportional to the imaginary part $k$ of the re- 
fractive index, provided conditions (i)-(v) above are satisfied. For clarity and simplicity, the case of steady illumination will be presented here; the applicability of similar considerations for pulsed synchrotron radiation will be shown in the Appendix. The light intensity decreases exponentialy with the distance from the entrance window:

$$
I(x)=I_{0} \exp \left[-\left(\alpha_{m}+\alpha_{p}\right) x\right],
$$

where $I_{0}$ is the number of photons entering the unit area of the sample in unit time, $I(x)$ their number at a distance $x$ from the sample-window interface, $\alpha_{m}$ the absorption coefficient due to the host, and $\alpha_{p}$ the same due to the impurity.

Since the current is proportional to the photon flux (according to experiment) and by conditions (i) and (ii) above only absorption by the impurity results in photoconduction, the concentration of free electrons $n(x)$ is

$$
n(x)=\tau \alpha_{p} I_{0} \exp \left[-\left(\alpha_{m}+\alpha_{p}\right) x\right],
$$

$\tau$ being the free-electron lifetime.

The total current $i$ between the electrodes is

$$
\begin{aligned}
i=e l \int_{0}^{\infty} E \mu n(x) d x & =e l\langle E \mu\rangle \int_{0}^{\infty} n(x) d x \\
& =e l \tau\langle E \mu\rangle I_{0} \frac{\alpha_{p}}{\alpha_{m}+\alpha_{p}} .
\end{aligned}
$$

Here $l$ is the effective length of the electrodes, $E$ the electric field strength, and $\mu$ the mobility. The integration can be taken to infinity if the penetration length of the light is smaller than the effective depth of the sample. In our experiment this depth is determined by the range of the electric field in the sample [condition (iii)]. $\langle E \mu\rangle$ is the average of $E \mu$ in the active volume of the sample; condition (iii) ensures that it should depend only weakly on the light intensity. For any given host density and impurity concentration $\langle E \mu\rangle$ can thus be regarded as roughly constant. Moreover, in a set of experiments with identical samples but different applied voltages we found that the current increased only very slightly on the voltage, demonstrating that the drift velocity was practically at its saturation values. ${ }^{4}$

It follows from Eq. (3) that $1 / i$ is proportional to $\alpha_{m} / \alpha_{p}$, provided $\alpha_{p} \ll \alpha_{m}$ [condition (iv)]. Since

$$
\alpha=\frac{4 \pi k}{\lambda}=\frac{2 k E_{p}}{\hbar c},
$$

where $\lambda$ is the wavelength, $\hbar$ Planck's constant divided by $2 \pi$, and $c$ the velocity of light in vacuum, one finds that under the above assumptions $1 / i E_{p}$ will be proportional to $k$, as stated above, if $\alpha_{p}$ is independent of the photon energy [condition (iv)].

It should be noted that a density or dopant concentration dependence of $\tau$ or of $\langle E \mu\rangle$ would not cause a distortion of any single spectrum, but it would introduce errors of the relative scalings of different spectra. However, all quantitative results of this paper are independent of the values of the scaling factor.

\section{B. The $n=2$ Mott-Wannier exciton}

The experimental results presented and the above considerations show that liquid xenon containing methyl iodide or toluene at high concentrations, with electrodes on the inner surface of the entrance window, is a system where the experimental method and proposed analysis are applicable. Well-resolved and reliable spectra were obtained in the vicinity of the $n=2$ Mott-Wannier exciton. We will analyze the $n=2$ exciton in terms of its peak position $E_{2}$, its height $B$, and its width $d$. Since the shape of this peak varies very little, $B d$ is roughly proportional to the area of the peak.

Figure 5 shows the height (normalized to the $E_{1}$ peak height at each density) and the width of this exciton at different liquid densities. It is seen that the height increases monotonically with density, while the width remains constant. In other words, the area under the absorption constant $k$ also increases monotonically with the density. We tried to interpret the latter results by means of simple statistical considerations, postulating that excitons can appear only in such regions, where the local density is high enough. ${ }^{8,10}$ According to this model, the rise of $B$ with the average density $\rho$ is due to the increase of the probability that in the sampling volume $V=V(\rho)$ there should be a prescribed local density $\rho^{\prime}$. As $V(\rho)$ we took the volume of a sphere the radius of which is equal to the Bohr radius [varying between 1.3 and $1.6 \mathrm{~nm}$ (de-
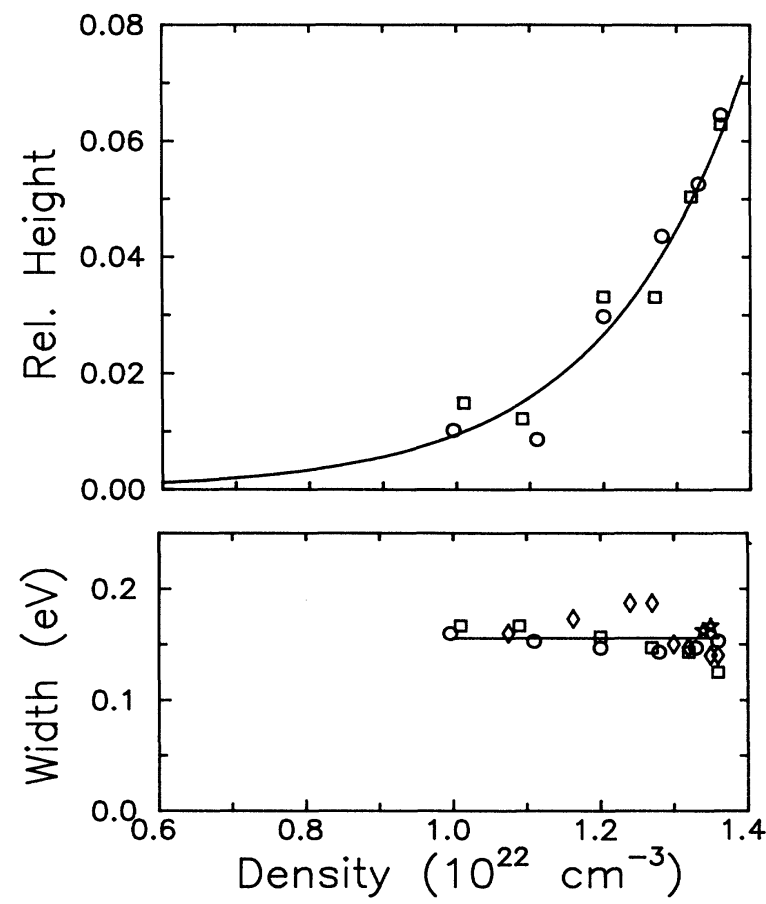

FIG. 5. Width (FWHM) and height of the $n=2$ exciton peak (normalized to the height of $E_{1}$ ) as a function of density. $\diamond$, Toluene $600 \mathrm{ppm}$; 5 , Toluene $6000 \mathrm{ppm}$; $\square, \mathrm{CH}_{3} \mathrm{I} 600 \mathrm{ppm}$; $O$, $\mathrm{CH}_{3} \mathrm{I} 1.1 \%$. The curve in the height vs density graph is an empirical exponential fit, $h=h_{0} \exp (\alpha \rho), h$ being the height and $\rho$ the number density. $h_{0}=5.36 \times 10^{-5}, \alpha=5.17 \times 10^{-22} \mathrm{~cm}^{-3}$. 
pending on $\rho$ )], of the $n=2$ exciton. With average density such a sphere contains between $\langle N\rangle=26$ and 72 atoms. Proceeding as in the papers quoted, ${ }^{8,10}$ we calculated the mean-square thermodynamic fluctuations $\left\langle(\Delta N)^{2}\right\rangle$ in each sample volume $V(\rho)$. Next the probability $w\left(\rho^{\prime}, V(\rho)\right)$ was calculated for having a local density equal to or larger than a fixed prescribed value $\rho^{\prime}$ in each of the volumes $V(\rho)$. However, for any reasonable choice of $\rho^{\prime}$ the rise of $w\left(\rho^{\prime}, V(\rho)\right)$ with $\rho$ was much steeper than that of $B$. Thus our primitive model, linking the appearance of the $n=2$ exciton with sufficient local density, is not adequate. By way of contrast, such a model was found to describe reasonably well the appearance of the $n=1$ "intermediate" exciton near the atomic line $6 s(3 / 2)_{1}^{0}$ in fluid xenon ${ }^{8}$ and krypton. ${ }^{10}$

Studying the positions of the $n=2$ excitons is of particular interest because of the relevance of this energy value to the binding energy $G$ and the reduced effective mass $m_{\text {exc }}$ of the exciton. Figure 6 shows the exciton peak positions $E_{2}$ as obtained in our experiments as a function of the density. It is immediately evident that $E_{2}$ is dependent not only on the density $\rho$, but also on the impurity concentration $c: E_{2}$ increases with decreasing $\rho$ and with increasing $c$. However, $E_{2}$ does not seem to be sensitive to the nature of the impurity, $\mathrm{CH}_{3} \mathrm{I}$ or $\mathrm{C}_{6} \mathrm{H}_{5} \mathrm{CH}_{3}$.

This apparent dependence of $E_{2}$ on the doping level is, in all probability, caused by the fact that the contribution $\alpha_{p}$ of the impurity to the absorption coefficient is not constant in this region and therefore condition (iv) (see above) is violated. No data seem to exist on the optical absorption caused by the methyl iodide impurity in xenon. We infer from the work by Messing, Raz, and

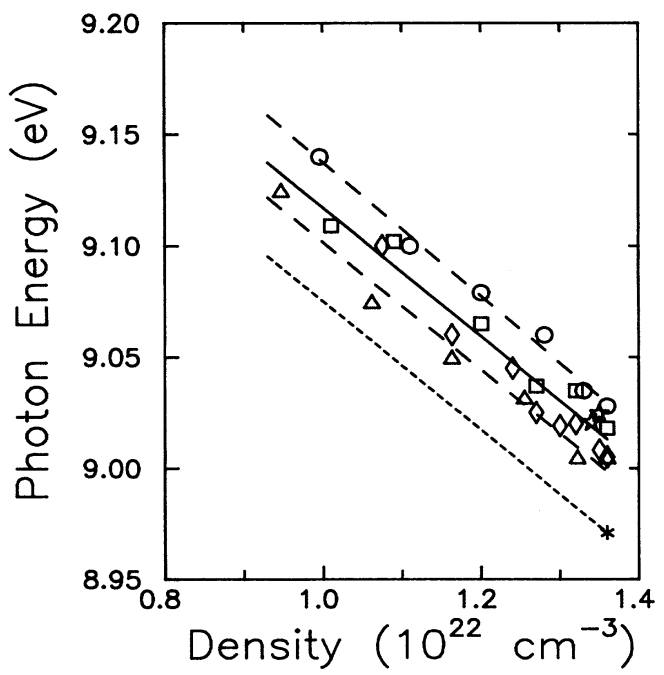

FIG. 6. The position of the $E_{2}$ peak as a function of density. Symbols as in Fig. 5 with the addition of $\triangle \mathrm{CH}_{3} \mathrm{I} 125 \mathrm{ppm}$. Lower - - , a linear regression fit to the data with $c=125$ ppm $\mathrm{CH}_{3} \mathrm{I}$; upper --- , the same with $c=1.1 \% \mathrm{CH}_{3} \mathrm{I}$; - a linear regression fit to all of the data; $\times, E_{2}$ from Ref. 14 ; ... extrapolation to zero impurity concentration; see text.
Jortner ${ }^{21}$ on $\mathrm{CH}_{3} \mathrm{I}$ in argon and krypton that $\alpha_{p}$ should be a decreasing function of $E_{p}$ in this region. Now, $1 / i$ is proportional to $1+\alpha_{m} / \alpha_{p}$ [Eq. (3)]. As a result (if, indeed, $\alpha_{p}$ decreases with increasing photon energy) the maximum of $1 / i$ will appear at a photon energy higher than that of $\alpha_{m}$. Similarly, the maximum of $1 / i E_{p}$ will appear above that of $k=\alpha_{m} c \hbar / 2 E_{p}$ [see Eq. (4)]. With the increase of impurity concentration this effect becomes more and more prominent, since the relative importance of $\alpha_{p}$ increases.

Whatever the reason for the observed shift, one has to extrapolate to zero impurity concentration the peak positions observed. This can be readily done, since Fig. 6 shows that concentration changes cause parallel translations of the same straight line. We used the result by Subtil et al. ${ }^{14}$ near the triple point to translate the line so as to correspond to zero concentration. Accordingly, we corrected our results by subtracting $0.041 \mathrm{eV}$ from the values of $E_{2}$ obtained by linear regression, $0.041 \mathrm{eV}$ being the difference between our value (9.012) and that of Subtil et al. ${ }^{14}(8.971 \mathrm{eV})$ at the number density of $13.63 \times 10^{21}$ $\mathrm{cm}^{-3}$. In other words, we claim that the correct $E_{2}$ values are those of the dotted line in Fig. 6.

It is of particular interest to extract the value of the electron effective mass from these results. Since we do not have enough data to check and apply quantum-defect theory ${ }^{22,23}$ properly, we base our considerations on the effective-mass model for Wannier-Mott excitons. Accordingly

$$
E_{n}=E_{\infty}-\frac{R m_{\mathrm{exc}}}{\epsilon^{2} n^{2} m} .
$$

$E_{\infty}$ is the limit of the excitonic series, $m_{\text {exc }}$ is the effective reduced exciton mass, $m$ is the free-electron mass

$$
\frac{m}{m_{\mathrm{exc}}}=m\left(\frac{1}{m_{e}}+\frac{1}{m_{h}}\right),
$$

$m_{e}$ and $m_{h}$ being the electron and hole effective masses, respectively. $\epsilon$ is the dielectric constant at the frequency corresponding to the binding energy of the exciton. We calculated $e$ using Sinnock's data ${ }^{24}$ for the triple-point liquid and using the Clausius-Mossotti relationship for other densities. Unfortunately, for the liquid we can use only the value of $E_{n}$ for $n=2$, since the radius of $E_{1}$ is much too small so that central cell corrections have to be applied $^{25,26}$ and in the liquid the $n>2$ states are not observed because of broadening. However, we can have a good estimate for $E_{\infty}$ using the intrinsic photoconductivity threshold $E_{\text {th }}$. While $E_{\text {th }}$ is close to $E_{\infty}$ it is always slightly lower: this is caused, in all probability, by the fact that excitonic levels with energies near to that of the bottom of the conduction band will ionize thermally and contribute to photoconduction. $E_{\infty}$ values were calculated from the experimentally observed $E_{\text {th }}$ values in the following manner. For the triple-point solid we have a very reliable value of $E_{\infty}$, obtained from excitonic series, namely, $9.303 \mathrm{eV} ;{ }^{14}$ at the same point $E_{\text {th }}=9.265 \mathrm{eV} .^{20}$ We assumed that at each temperature $E_{\infty}-E_{\text {th }}=\alpha T, \alpha$ being a proportionality constant, obtainable from the 


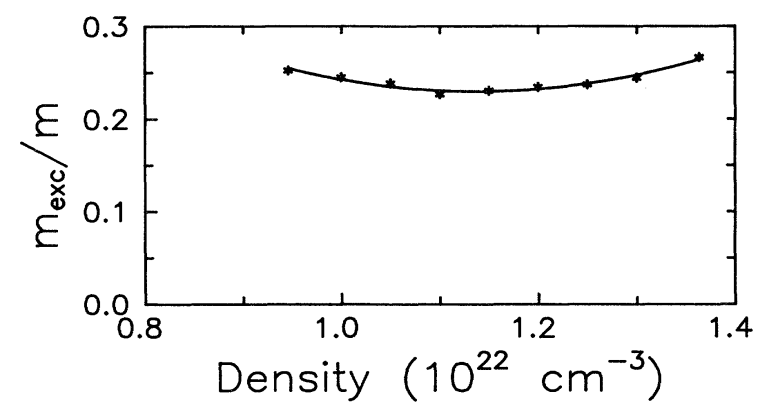

FIG. 7. The reduced effective exciton mass $m_{\text {exc }}$ divided by the free-electron mass $m$ as a function of density. The curve is an empirical parabolic fit.

difference of $E_{\infty}-E_{\text {th }}$ at the triple point. After applying such a correction we could use $E_{\text {th }}$ values determined by Reininger et al. in a broad range of densities. ${ }^{20}$

By this procedure the value of $m_{\text {exc }}$ was calculated from Eqs. (5) and (6). The results presented in Fig. 7 show that $m_{\text {exc }} / m \approx 0.25$ in the whole density range, with a shallow minimum at about $\rho=11 \times 10^{21} \mathrm{~cm}^{-3}$. It is noteworthy that this minimum is at about the same density as the minimum of $V_{0}$ (Ref. 15) and the maximum of the zero-field electron mobility. ${ }^{4}$ However, one should treat these results with some caution, since the depth of the minimum is smaller than the limits of the probable error (about 0.03; the error is not evident from Fig. 6, since we used least-squares fitted values of $E_{2}$ and smoothed values of $E_{\mathrm{th}}$ ). One can estimate the hole effective mass as $2.1 \mathrm{~m}$ in this density range; ${ }^{27}$ it follows from (6) that the effective electron mass is about $0.28 \mathrm{~m}$. Recent calculations of $m_{e}$ in rare-gas liquids by a method proposed by Bardeen in the framework of the WignerSeitz calculation give for $\mathrm{Xe}$ near the triple point $m_{e}=0.40 .^{28}$ According to these calculations, ${ }^{28} m_{e}$ increases monotonically as the density decreases.

To the best of our knowledge, this is the first determination of the electron effective mass in any liquid for a broad range of densities.

\section{CONCLUSIONS}

It has been demonstrated that impurity photoconduction spectra can be readily analyzed to obtain a good representation of the host absorption spectrum. In particular, the width, height and position of the $n=2$ MottWannier exciton in liquid xenon have been analyzed as a function of number density in a wide range of densities. From these results, combined with intrinsic photoconduction data, the exciton and electron effective masses were obtained and compared with existing theoretical calculations.

\section{ACKNOWLEDGMENTS}

This research was supported by the United States-Israel Binational Science Foundation (BSF) Jerusalem, Israel, Grants No. 85-0080 and No. 88-00107.

\section{APPENDIX}

The applicability of the $1 / i E_{p}$ versus $E_{p}$ representation for excitation by synchrotron radiation will be discussed here. In our experiments, the synchrotron radiation consisted of a sequence of subnanosecond light pulses, having the duration $t_{0}=0.13 \mathrm{nsec}$ and separated by $t_{1}=240 \mathrm{nsec}$ time intervals. The current was measured at each photon energy during a time $t_{2}$, which was chosen, in order to obtain a good signal-to-noise ratio, either as 1 or $2 \mathrm{sec}$, depending on the experiment. Without going into any detail about the dynamics we can state that the considerations given at the beginning of the Discussion are valid, provided we deal with time average of all variables over times of the order of $t_{2}$. However, those considerations involve the essential requirement [condition (v)] that the electron lifetime stays constant. In order to check whether this condition is indeed fulfilled, one has to discuss to some extent the actual processes taking place during times much shorter than $t_{2}$.

After monochromatization, about $10^{4}$ photons per pulse reach the sample and may generate electron-ion pairs. The electrons drift towards the anode and, at a much lower rate, the positive ions drift towards the cathode. Most of the positive ions cannot reach the cathode during the time interval between consecutive pulses, $t_{1}=240$ nsec. As a consequence, after a number of light pulses a positive charge will build up near the cathode. In the central region of the sample the field will become appreciably smaller than the average one because of the potential drop in the space-charge region.

After the termination of the light pulse the time dependence of the electron population is

$$
\frac{\partial n}{\partial t}=-\beta n(n+N)-\frac{n}{\tau_{n}} \text {. }
$$

In this equation $n$ is the density of electron-ion pairs created by the preceding light pulse and $N$ is the density of positive ions that remained from previous pulses. The probability for electron-ion recombination is determined by $\beta ; 1 / \tau_{n}$ is the probability that an electron is captured by a neutral impurity. Excitation transfer processes are neglected here. We note that $n=n(x, z, t)$ and $N=N(x, z, t)$, where $x$ is the coordinate perpendicular to the window and $z$ is parallel to the window and perpendicular to the electrodes.

By integration, assuming $N$ and $\tau_{n}$ are time independent, we get

$$
\begin{aligned}
n & =\frac{n_{0}\left(\beta N \tau_{n}+1\right) \exp (-t / \tau)}{1+\left(n_{0}+N\right) \beta \tau_{n}-n_{0} \beta \tau_{n} \exp (-t / \tau)} \\
& \approx n_{0} \exp (-t / \tau),
\end{aligned}
$$

where $1 / \tau=\beta N+1 / \tau_{n}$ and $n_{0}$ is the electron concentration at the end of the synchrotron light pulse. Obviously $n_{0} \ll N$; this justifies the approximation in Eq. (A2). One may safely assume that $t_{0}<<\tau$, since otherwise the current would be minuscule, and that $\tau<t_{1}$, since the (unintentional) impurity concentration of the xenon gas used was about $5 \mathrm{ppm}$. We note that $N$ is not truly time 
independent, but it varies much more slowly then $n$, since the drift of the ions is much slower than that of the electrons. In order to calculate the current precisely, the space-charge distributions ought to be accurately known, and the variation of the drift velocity with the field ought to be taken into account. Obviously, specially designed experiments would be needed in order to obtain enough data for such calculations. ${ }^{29}$ However, we obtain a very significant simplification if we take into account that the positive space charge builds up always to about the same final distribution, independently of the photon energy. This is so since the ion currents are space-charge limited-the final charge distribution is determined by the rate of neutralization at the negative electrode and Poisson's equation. The photon energy determines the number of free electrons created per pulse and this number does influence, of course, the time $\tau_{s}$ needed for the buildup of the space charge. Since the ion motion is much slower than that of the electrons, $\tau<<\tau_{s}$ and $t_{1} \ll<\tau_{s}$. In fact, the latter inequality will be satisfied with a factor of a thousand or more. However, even so $\tau_{s}$ will be hundreds of times shorter than the sampling time $t_{2}$ $(1-2 \mathrm{sec})$. Thus, at any given point $(x, z)$ the electrons will "see" the same ion density during almost all the sampling time. It follows that $1 / \tau=1 / \tau_{n}+\beta N$ is, to a very good approximation, independent of the photon energy, as postulated in condition $(\mathrm{v})$.

For a detailed solution of the problem, one would have to know the dependencies of $n, \tau_{n}, N$, and the field $E$ on the coordinate $z$. Since these cannot be determined by the present experiments, we replace $n(x, z)$ by $n(x)$ defined as an appropriate weighted average of $n(x, z)$ that carries the same current as the distribution $n(x, z)$ (at constant $x)$. Then $\tau_{n}(x)$ and $N(x)$ are defined as $\tau_{n}(x, z)$ and $N(x, z)$, respectively, at a point $(x, z)$ where $n(x)=n(x, z)$. The justification of this procedure is again in the stable final space-charge distribution.

The rest of the calculation is straightforward. The free-electron concentration $n_{0}$ at the termination of a light pulse is given by

$$
n_{0} \approx \alpha_{p} t_{0} I_{0} \exp \left[-\left(\alpha_{m}+\alpha_{p}\right) x\right],
$$

since $\tau \gg t_{0}$. Here $I_{0}$ is, as in Eq. (2), the number of photons incident (during the pulse) in unit time on unit area. To calculate the total current, note that the time average of $n$ is $n_{0} \tau / t_{1}$, since $t_{1} \gg \tau \gg t_{0}$ [see Eqs. (A2) and (A3)]. Then the total current is obtained by integration over $x$, as in Eq. (3):

$i=e l \int_{0}^{\infty} E \mu n(x) d x=e l \tau\langle E \mu\rangle \frac{t_{0}}{t_{1}} I_{0} \frac{\alpha_{p}}{\alpha_{m}+\alpha_{p}}$.

Since this equation is identical-apart from the constant factor $t_{0} / t_{1}$ on the right-hand side-to Eq. (3) in the Discussion, the applicability of the scheme for synchrotron radiation excitation follows directly.
${ }^{1}$ B. Halpern, J. Lekner, S. A. Rice, and R. Gomer, Phys. Rev. 156, 351 (1967).

${ }^{2}$ L. S. Miller, S. Howe, and W. E. Spear, Phys. Rev. 166, 871 (1968).

${ }^{3}$ J. A. Jahnke, L. Meyer, and S. A. Rice, Phys. Rev. A 3, 734 (1971).

${ }^{4}$ S. S. S. Huang and G. R. Freeman, J. Chem. Phys. 68, 1355 (1978).

${ }^{5}$ S. S. S. Huang and G. R. Freeman, Phys. Rev. A 24, 714 (1981).

${ }^{6}$ D. Beaglehole, Phys. Rev. Lett. 15, 207 (1965).

${ }^{7}$ U. Asaf and I. T. Steinberger, Phys. Lett. 34A, 207 (1971).

${ }^{8}$ P. Laporte and I. T. Seinberger, Phys. Rev. A 15, 2538 (1988).

${ }^{9}$ P. Laporte, J. L. Subtil, U. Asaf, I. T. Steinberger, and S. Wind, Phys. Rev. Lett. 45, 2138 (1980).

${ }^{10}$ P. Laporte, J. L. Subtil, R. Reininger, V. Saile, S. Bernstorff, and I. T. Steinberger, Phys. Rev. B 35, 6270 (1987).

${ }^{11}$ J. Jortner, in Vacuum Ultraviolet Radiation Physics, edited by E. E. Koch, R. Haensel, and C. Kunz (Pergamon-Vieweg, Braunschweig, 1974), p. 263.

${ }^{12}$ I. Messing, B. Raz, and J. Jortner, Chem. Phys. 23, 23 (1977).

${ }^{13}$ N. Schwentner, E. E. Koch, and J. Jortner, Electronic Excitations in Condensed Rare Gases (Springer-Verlag, Berlin, 1985).

${ }^{14}$ J. L. Subtil, P. Laporte, R. Reininger, and V. Saile, Phys. Status Solidi B 143, 783 (1987).

${ }^{15}$ R. Reininger, U. Asaf and I. T. Steinberger, Chem. Phys. Lett. 90, 287 (1982).

${ }^{16}$ V. Saile, P. Gürtler, E. E. Koch, A. Kozevnikov, M. Skibowski, and W. Steinmann, Appl. Opt. 15, 2559 (1976).
${ }^{17}$ UHU Endfest 200, manufactured by Lingner + Fischer, 7580 Buhl, Germany.

${ }^{18}$ W. B. Streett, L. S. Sagan, and L. A. K. Staveley, J. Chem. Thermodyn. 5, 633 (1973).

${ }^{19}$ I. T. Steinberger, U. Asaf, G. Ascarelli, R. Reininger, G. Reisfield, and M. Reshotko, Phys. Rev. A 42, 3135 (1990).

${ }^{20}$ R. Reininger, U. Asaf, I. T. Steinberger, V. Saile, and P. Laporte, Phys. Rev. B 28, 3193 (1983); R. Reininger, Ph.D. thesis, The Hebrew University of Jerusalem, Jerusalem, 1982 (unpublished).

${ }^{21}$ I. Messing, B. Raz and J. Jortner, Chem. Phys. 25, 55 (1977).

${ }^{22}$ L. Resca, R. Resta, and S. Rodriguez, Phys. Rev. B 18, 696 (1978); L. Resca and R. Resta, ibid. 19, 1683 (1979); R. Resta, Phys. Status Solidi B 86, 627 (1978).

${ }^{23}$ V. Saile, R. Reininger, P. Laporte, I. T. Steinberger, and G. L. Findley, Phys. Rev. B 37, 10901 (1988).

${ }^{24}$ A. C. Sinnock, J. Phys. C 13, 2375 (1980).

25J. Hermanson and J. C. Phillips, Phys. Rev. 150, 652 (1966).

26J. Hermanson, Phys. Rev. 150, 660 (1966).

${ }^{27}$ U. Rössler, in Rare Gas Solids, edited by M. L. Klein and J. A. Venables (Academic, London, 1976), p. 505.

${ }^{28}$ B. Plenkiewicz (private communication); B. Plenkiewicz, P. Plenkiewicz, and J.-P. Jay Gérin (unpublished).

${ }^{29}$ The case is probably an intermediate one between free and ambipolar diffusion and thus the positive ion mobility may play an important role. See, e.g., W. P. Allis, in Elektronen Emission, Gasentladungen I, edited by S. Flügge, Handbuch der Physik Vol. XXI (Springer-Verlag, Berlin, 1956 ), p. 383. 